\title{
De Helsinki a Fortaleza: una Declaración desangrada
}

\section{Resumen}

La Declaración de Helsinki 1964 ha sido sometida a numerosas revisiones y enmiendas, la más reciente siendo Fortaleza (2013), una frecuencia considerada necesaria dado los avances de la medicina contemporánea, pero también criticada por restarle estabilidad y autoridad al documento. La versión de Edimburgo (2000) enfatizó la tolerancia al uso de placebos y restó apoyo de los beneficios post-estudio para los probandos y la comunidad huésped, pese a los esfuerzos de Argentina y Brasil por robustecer la protección de las personas incorporadas a los estudios. Queda aceptado - de hecho - el doble estándar en la ética de investigación que debilita la Declaración como normativa ética de la investigación con seres humanos. La dominancia acentuada de los intereses corporativos de investigadores y patrocinadores sugiere que la bioética latinoamericana debiera desarrollar su propio documento normativo, en respeto a nuestra realidad social, y enfocado a la protección de las comunidades de la región.

Palabras-clave: Declaración de Helsinki. Ética en investigación. Protección.

\section{Resumo}

\section{De Helsinki à Fortaleza: uma declaração dessangrada}

A Declaração de Helsinki de 1964 vem sendo submetida a numerosas revisões e emendas, sendo a mais recente a de Fortaleza (2013). A frequência dessas reformulações tem sido considerada necessária, dados os avanços da medicina contemporânea, mas também criticada por conferir pouca estabilidade e autoridade ao documento. A versão de Edimburgo (2000) marcou a política de tolerância ao uso de placebo e de escasso apoio aos benefícios pós-estudo aos sujeitos e à comunidade, desestimulando os esforços da Argentina e Brasil para reforçar a proteção aos participantes dos estudos. Se aceita assim - de fato - o duplo standart em ética em pesquisa, que de forma progressiva debilita a Declaração como normativa ética para a pesquisa envolvendo seres humanos. A dominação cada vez mais acentuada de interesses corporativos de investigadores e patrocinadores sugere que a bioética latino-americana deve desenvolver seu próprio documento normativo, em respeito a nossa realidade social, voltado a proteção às comunidades da região.

Palavras-chave: Declaração de Helsinki. Ética em pesquisa. Proteção.

\section{Abstract \\ From Helsinki to Fortaleza: a bled declaration}

The original Declaration of Helsinki (1964) has been subjected to numerous revisions and reformulations, supposedly necessary to keep apace with medical progress, but in fact leading to its loss of stability and authority. The Edinburgh 2000 revision made provisions for increased tolerance in the use of placebos, and loss of commitment to assure post-investigational benefits to individuals and communities involved. In spite of Argentina's and Brazil's manifest opposition, a double standard for research ethics became a de facto reality which has increasingly weakened the Declaration. Corporative interests of major stakeholders -researchers and sponsors- have been strongly supported as the Declaration becomes less protective of individuals and communities involved. It is therefore suggested that Latin American bioethicists would be well advised to develop a regionally pertinent normative in accordance with our social reality and the need of protecting our population.

Key words: Declaration of Helsinki. Ethics, research. Protection.

Doutor mkottow@med.uchile.cl - Universidad de Chile, Santiago, Chile.

\section{Correspondência}

José Miguel de la Barra 521 Depto. 4-B 5602 26380614. Santiago, Chile.

Declara não haver conflito de interesse. 
Se repite con obstinada frecuencia que la $D e$ claración de Helsinki (1964) se gestó como la indignada respuesta a las atrocidades cometidas en el régimen fascista alemán (1933-1945) disfrazadas bajo el eufemismo "experimentación" biológica. La misma justificación recibe el Código de Núremberg el cual, efectivamente, fue una reacción inmediata a los horrores de la guerra, los campos de concentración, las sevicias y las torturas médicas. Los científicos de la post-guerra protestaron, y con razón, que no cabe homologar la condena de la tortura con la ética de la investigación. Así se explica que Núremberg sea un Código único, inamovible, determinado, mientras la Declaración, presentada casi 20 años después, es propositiva, modificable y, de hecho, revisada en siete ocasiones, la más reciente, pero con certeza no la última, siendo Fortaleza (2013).

La influencia de la Declaración ha ido decreciendo, mayormente lesionada por la FDA que se desafilió de ella y decidió ceñirse por la GCP (Guidelines for Good Clinical Practice = Guías de Buenas Prácticas Clínicas). El punto de corte fue la revisión de Edimburgo 2000, tenazmente resistida por las delegaciones de Argentina y Brasil ${ }^{1}$, que fue doblegada por propuestas de un uso más flexible y permisivo de placebos en investigación con seres humanos y a una indeterminación de beneficios postestudio para los participantes.

En 2008, coincidente con la en aquel momento reciente revisión (Seúl, 2008), la FDA (Food and Drug Administration) oficialmente negó su apoyo a la Declaración y se acogió a la GPC que, si bien rinde pleitesía a Helsinki, es más proclive a respetar intereses corporativos de la industria farmacéutica y de los investigadores, en desmedro de la máxima protección de probandos. La postura de la FDA fue criticada como una maniobra de dudoso valor ético por su tolerancia del uso de placebos, que nos llevaría a la paradójica conclusión de que sería ético hacer investigaciones en África, Sudamérica o Indonesia, y en contraposición a las normas éticas en EE.UU., Europa o Japón ${ }^{2}$ e, un ejemplo decepcionante para muchas naciones ${ }^{3}$.

La revisión de la Declaración de Helsinki en Seúl (2008) fue seguida con gran atención y en general criticada por robustecer posiciones éticamente condenables que terminaron por delinear $y$ ratificar la ética del "doble estándar" 4: uno "aspiracional" o de máximos aplicado en los países de mayor desarrollo económico, y uno pragmático o de contexto que relativiza el rigor ético para países pobres, corruptibles, menos educados, de población "vulnerable" en el sentido de ser "incapaz de defen- der sus propios intereses" 5,6. La Declaración pierde influencia por la progresiva debilidad de lo que es, o debiera ser su propósito nuclear: recomendar el bienestar de los sujetos de investigación [que] deben ser siempre protegidos y no sufrir la subordinación a intereses de terceros ${ }^{7}$.

Se lidia con la paradoja que los mismos investigadores que denigran la Declaración invierten ingentes esfuerzos por llevarla a revisiones cada vez menos favorables para los probandos individuales y las comunidades huésped, fortaleciendo, en cambio, el apoyo a patrocinadores e investigadores vinculados a corporaciones transnacionales e instituciones poderosas como el NIH (National Institutes of Health) ${ }^{8}$. Helsinki queda diluida y denigrada, distorsionada por el establecimiento fáctico de una [bio]ética de investigación con seres humanos basada en protocolos que en sus países de origen serían éticamente inaceptables.

En diversas opiniones vertidas frente a la inminente revisión de 2013, fueron aducidos los mismos argumentos que aún perduran insolutos: el uso de placebos, la administración al grupo control de los mejores medios médicos existentes vs. los localmente disponibles, el grado de compromiso post-estudio, la conveniencia de que investigador y médico tratante fuese una misma persona, el utilitarismo como pertinente a una ética de investigación en general, a la Declaración de Helsinki en particular.

\section{Una extinción lamentable: estudios clínicos terapéuticos/no terapéuticos}

La Declaración de Helsinki (1964) consta de una página, cuyo cuarto párrafo dice: In the field of clinical research a fundamental distinction must be recognized between clinical research in which the aim is essentially therapeutic for a patient, and clinical research the essential object of which is purely scientific and without therapeutic value to the persons subjected to the research ${ }^{9}$.

El médico, especifica el documento, podrá combinar investigación con cuidados profesionales, a objeto de ganar nuevo conocimiento médico, solo en la medida que la investigación clínica se justifique por su valor terapéutico para el paciente ${ }^{10}$, una distinción ya enfáticamente requerida por Hans Jonas 20 años antes ${ }^{11}$. La Declaración fue sometida a cinco revisiones a lo largo de 36 años, la de Edinburgo (2000) precedida de una larga polémica sobre la conveniencia, la urgencia según algunos, y la necesidad de una revisión a fondo, frente a la cautela pro- 
piciada por otros preocupados que estas revisiones podrían inadecuadamente llevar una desviación hacia estándares de investigación centrados en eficiencia y debilitar los principios morales del investigador comprometido con el sujeto de investigación y la justa asignación de beneficios y cargas $^{12}$. La distinción entre estudio terapéutico y no terapéutico terminó por desaparecer, asfixiada por el argumento de que [L]a clase de actividades cubiertas por el término "investigación terapéutica" es a su vez problemática por cuanto todos los ensayos clínicos de agentes terapéuticos incluyen algunos componentes que pueden ser terapéuticos (o intencionados como tales) y otros que son claramente no terapéuticos ${ }^{13}$.

La extinción de la originaria y explícita distinción entre ensayos clínicos terapéuticos y no terapéuticos, llevó a dos conclusiones que claramente aumentan la desprotección de los pacientes incorporados como probandos: 1) La "posición de similitud" que considera la ética de la investigación clínica como meramente un caso especial o de aplicación de la ética clínica pertinente a la práctica médica, y la posición de diferencia, según la cual la investigación y la práctica clínica son actividades distintas con diversas metas - acopio de conocimientos para beneficiar a futuros pacientes vs. el beneficio terapéutico para el paciente individual ${ }^{14}$. En otra publicación, estos mismos autores, propiciando la diferencia entre ética clínica y ética de la investigación, reconocen que en independencia de las motivaciones del investigador, los pacientes voluntarios están en riesgo de ver comprometido su bienestar en el transcurso de una investigación cientifica ${ }^{15}$.

La disputa es ocultada al recurrir a un fraseo como el del acápite 16 de la revisión de Fortaleza en 2013: Investigación médica que incorpora sujetos humanos solo podrá realizarse si la importancia del objetivo es mayor que los riesgos y cargas para los sujetos de investigación ${ }^{16}$. Por diversas razones, es una propuesta vacía, sujeta a interpretaciones arbitrarias sobre lo que es un objetivo importante, y la evaluación de riesgos y cargas, que de ningún modo asegura la protección de pacientes involucrados en estudios clínicos. La revisión de Fortaleza es el réquiem a la Declaración de Helsinki 1964.

\section{La revisión de Fortaleza}

¿Por qué se sustituyó el término "poblaciones" prefiriendo hablar de "grupos"? El vocabulario deóntico (should, must, may) tiene un uso fluctuante y al parecer arbitrario. En la sección "Informed consent" se reemplaza "competent" por "capable of giving informed consent": en una lectura tal vez hipercrítica podría argumentarse que la "competencia" es objetivamente evaluable, siendo más cómodo hablar de "la capacidad de", que se presta a una apreciación más subjetiva y sesgada.

La referencia a placebos (ahora $n^{\circ} 33$ ) continúa siendo favorable a una interpretación por los investigadores; el sutil cambio de "any risk of serious or irreversible harm" por "additional risk of serious or irreversible harm as a result of not receiving the best proven intervention" ${ }^{16}$ genera una mayor indeterminación al hablar de riesgos - que son potenciales- en vez de reconocer efectos secundarios lesivos realmente ocurridos. Queda manifiesta, además, la dificultad de demostrar la causalidad directa del daño por la omisión de los mejores medios médicos comprobados (debiera decir existentes).

El otro punto de contención referido a "provisiones post-estudio" ahora se refiere a las "provisiones previas al estudio para todos los participantes" ${ }^{16}$. No hay una Declaración vinculante de que los probandos recibirían los beneficios que médicamente les son necesarios, tan solo es validado un vago compromiso con participantes que aún requieren una intervención identificable en el estudio $\left(n^{\circ} 34\right)^{16}$.

\section{Revisando las revisiones}

Inconcluso el debate y sus insatisfactorios resultados, Edimburgo (2000) fueron seguida de dos notas aclaratorias (Washington 2002, Tokio 2004), y dos revisiones (Seúl 2008, Fortaleza 2013) y diversas notas aclaratorias, con una periodicidad de un lustro, señalando así que la Declaración se encuentra permanentemente en la mira de críticas y polémicas:

The frequency with which the Declaration of Helsinki has been revised - about every 6 years - is itself a problem... This process of revision raises doubts about whether the Declaration's guidance is really well reasoned and authoritative; it encourages researchers not to take the Declaration seriously. Genuine ethical obligations do not change every few years ${ }^{17}$.

Apenas proclamada la primera Declaración de Helsinki, fue reconocida como un documento que seguirá siendo controversial que, a lo muy menos y por de pronto, demuestra la preocupación de la profesión médica mundial por los temas éticos involucrados [en la experimentación humana] ${ }^{9}$. Es un reconocimiento de ineficacia y una invitación a revisiones que han resultado ser interminables. Investi- 
gadores empeñados en fortalecer la posición de una ética de investigación que reemplace la ética clínica cuando un paciente es reclutado como probando, han insistido en la necesidad de revisar aspectos "defectuosos" de la Declaración ${ }^{18}$.

Al mismo tiempo, la Asociación Médica Mundial (AMM) reconoció que los estudios sobre el uso de zidovudine para prevenir la transmisión perinatal de infección por VIH, estaban creando presión para corregir los principios de investigación involucrando sujetos humanos ${ }^{19}$. Las revisiones propuestas debilitan el principio del compromiso moral de investigador con el sujeto de investigación y reducen la protección de derechos de [dichos] sujetos. La eficiencia utilitarista, junto a los valores del Mercado, es más prominente, y estos valores son aplicados en cualquier contexto socioeconómico ${ }^{19}$.

Es posible que la Declaración requiera modernización...pero ha de mantener sus metas (de proteger a sujetos humanos) y sentar un estándar ideal ${ }^{20}$; opinión que es rebatida con el argumento que los códigos y guías de ética deben ser aseveraciones muy prácticas de lo que creemos que los investigadores pueden y deben hacer hoy en día ${ }^{21}$. $\mathrm{Y}$ han de ser utilitarios, por cuanto el utilitarismo es simplemente un método de análisis ético que evalúa la corrección ética de conductas humanas en relación a las consecuencias anticipadas ${ }^{21}$.

Sin ánimo de redundar, es ésta una postura excesivamente utilitarista del utilitarismo, en el sentido de obviar toda mención de la definición y distribución de lo útil y de los costos que significa obtenerlo. En suma, la reciente actualización de la Declaración de Helsinki muestra un padrón similar a las otras, resolviendo pocos problemas suscitados en versiones anteriores, presentando variaciones semánticas menores sin aclarar los puntos polémicos, prosiguiendo en debilitar la protección de probandos, individuales y colectivos, y asfixiando a quienes prosiguen en la brega por asegurar su autonomía.

\section{Árboles derribados, bosques quemados}

El proceso de construcción/deconstrucción de la Declaración de Helsinki es un desolado campo de disputas desgastantes y batallas perdidas en que han naufragado los esfuerzos por normar la [bio] ética de la investigación con seres humanos en forma respetuosa de los más débiles y regulativa de los insaciables intereses corporativos. La AMM se precia de revisar [sus] documentos en forma regular, modificándolos cuando parezca apropiado a fin de enfrentar desafíos actuales y futuros. La Declaración de Helsinki es, por lo tanto, un documento vivo que ha sido adaptado a lo largo del tiempo en respuesta a desarrollos en la investigación médica ${ }^{22}$. Más que justificar flexibilidad y actualidad, queda la impresión que la frecuencia de estas revisiones obedece a presiones externas y denota un proceso de debilidad y heteronomía que no responden a nuevos desarrollos de la biomedicina, como aducido, sino a reformulaciones de asuntos que siguen sometidos a polémica y a tensiones que se resuelven inadecuada y provisoriamente.

En el artículo citado, cuyos autores son miembros del grupo de trabajo World Medical Association Declaration of Helsinki, se insiste en el proceso de consulta pública para comentar el borrador de la versión revisada y la convocatoria a eminentes bioeticistas, cuyas recomendaciones, sin embargo, son muy parcialmente adoptadas, al punto de retener muchas fallas-y contradicciones-...y agregarfallas nuevas ${ }^{23}$. Como resultado, la nueva Declaración probablemente lleve a nuevas discusiones por ciertos patrocinadores de investigación... [llevando] a importantes stakeholders [grupos de intereses] a adherir preferentemente a versiones anteriores de la Declaración, a fin de obviar el enfrentamiento con ciertas provisiones incluidas en versiones más recientes ${ }^{24}$.

Inquietante es el comentario publicado apenas un mes después de presentada la revisión Fortaleza 2013, cuyo subtítulo es Progress but Many Remaining Challenges (Progreso, pero muchos desafíos restantes) - según el cual faltaría reconocer la posibilidad de obviar el consentimiento informado en ciertos casos de investigación con adultos competentes, desarrollar más asesoría para obtener consentimientos "amplios" para uso futuro de material biológico, destacar que son obstáculos limitantes para la ciencia los esfuerzos por proteger la investigación en individuos incapaces de consentir a estudios no relacionados con su incapacidad, así como serían inconsistentes las oposiciones a estudios "no benéficos" cuando los riesgos netos a los intereses del participante son bajos y los beneficios para la sociedad suficientemente grandes ${ }^{25}$. Continúa el texto señalando que la Declaración es confusa y errada en lo que a vulnerabilidad y protección adecuada se refiere ${ }^{25}$ y sugiere la necesidad de revisar el párrafo 34 referente a beneficios post-estudio y el párrafo 33 referente a placebos. Ante una agenda como la propuesta, es de temer que una nueva y pronta revisión desmedre aún más la autonomía y protección de probandos y de poblaciones declaradas "vulnerables." 
En suma, el estatuto de la Declaración es cambiante, su estatus va en declinación, la reacción inmediata a la más reciente revisión reconoce insolutos los problemas pendientes y agrega nuevos puntos de debate que, siendo muy sensibles, necesariamente llevarán a prolongadas discusiones y a continuar el proceso de desestabilización de la Declaración, condicionando y neutralizando cada vez más los esfuerzos por proteger a los probandos y subordinar, como debiera ser, la ética clínica a la ética de investigación, dando por aceptado, al menos de facto, el doble estándar de la ética de investigaciones clínicas.

\section{Traducción al español}

Cuarenta y ocho horas después de publicada la reciente revisión de la Declaración, aparece la traducción al español, causando la inmediata reacción alarmada de Luis Justo, quien envía un breve mensaje electrónico titulado "Cuidado Latinoamérica: Grave error de traducción de Helsinki 2013 al español": When no proven intervention exists que es tergiversado al español como "Cuando existe una intervención no probada". Siguiendo su sugerencia que la versión español "está mal traducida, muy mal traducida", es posible encontrar, efectivamente, por lo menos 50 trechos traducidos que son imprecisos o incorrectos, como por ejemplo: developed = "promulgado" en vez de desarrollado, each paragraph = "un párrafo" en vez de cada párrafo, burden= "costos" en vez de cargas, sponsors = "auspiciadores", en vez de patrocinadores; patients who serve as research subjects = "pacientes que forman parte de la investigación", en vez de pacientes que sirven como sujetos de investigación; repositories = "depósitos similares", en vez de repositorios; individual research subjects = "la persona que participa en la investigación", en vez de sujetos individuales de investigación.

No corresponde aquí evaluar acaso la traducción comete errores banales o interpretaciones eufemísticas, sino solo mostrar arbitrariedades semánticas cuya corrección puede diferir de la aquí sugerida, pero no desmiente que la versión española es impropia. El documento original está lejos de ser un primor estilístico, pero la traducción es aún más defectuosa en ese aspecto.

\section{Consideraciones finales}

El presente texto no tiene la intención de debatir sobre el contenido conceptual de la reciente Declaración de Helsinki ni subrayar la decepción que sienten los bioeticistas latinoamericanos con cada nueva revisión. El propósito es más bien esclarecer los mecanismos retóricos e interesados que están influyendo sobre este documento, al punto de llevarlo a un ya consolidado desprestigio que afecta seriamente el trabajo de la bioética regional y complica las labores de los Comités de [bio]ética de la investigación con seres humanos. Las instituciones bioéticas, incluyendo la docencia, dejan de poder confiar en la Declaración de Helsinki, tienen cada vez menos influencia para rectificarla, y deben recelar de otras guías de ética de la investigación que declaran estar basadas en Helsinki.

La conclusión, que ha sido insinuada en diversas ocasiones lamentablemente sin mayor trascendencia, es que la bioética latinoamericana haría bien en abandonar la estéril polémica en torno a la $D e$ claración, y abocarse seriamente a construir su propio instrumento de guía para la investigación clínica, respetuosa y relevante a la realidad social en que viven nuestras naciones, creando una inmunidad potente contra la colonización por intereses corporativos y una bioética "utilitarista" que se subordina a ellos. Debemos tomar en serio las tareas de asesoría y orientación en defensa y protección de nuestra población, desde una visión comprometida con las necesidades de la región ${ }^{26,27}$.

\section{Referências}

1. Editorial. Dismantling the Helsinki Declaration. Canadian Medical Association Journal. 2003; 169(10): 1-2.

2. Camporesi S. The FDA decision to shelve the Helsinki Declaration: ethical considerations. Ecancermedicalscience. 2008; Doi 10.3332/eCMS.2008.LTR76. (acesso 2 jan. 2014).

3. Kimmelman J, Weijer C, Meslin RM. Helsinki discords: FDA, ethics, and international drug trials. The Lancet. 2009 jan. 3; 373:13-14.

4. Pfeiffer ML, editora. Bioética ¿estrategia de dominación para América Latina? Mar del Plata: Ediciones Suárez; 2004.

5. Council for International Organizations of Medical Sciences. Pautas éticas internacionales para la investigación biomédica en seres humanos. Ginebra: Cioms; 2002. 
6. Macklin R. Double standards in medical research in developing countries. Cambridge: Cambridge University Press; 2004.

7. Giordano S. The 2008 Declaration of Helsinki: some reflections. Journal of Medical Ethics. 2010; 36: 598-603.

8. Rothman DJ. The shame of medical research. The New York Review of Books. 2000; 47 (19): 60-4.

9. Gilder S. World Medical Association meets in Helsinki. Brit. Med. J. 1964; 2: 299-300.

10. Ethical Committee of the World Medical Association. Draft code of ethics on human experimentation. BMJ. 1964;2:177-80.

11. Jonas H. Philosophical reflections on experimenting with human subjects. In: Freund PA, editor. Experimentation with human subjects. New York: George Braziller; 1969. p. 1-31.

12. Brennan TA. Proposed revisions to the Declaration of Helsinki - will they weaken the ethical principles underlying human research. The New England Journal of Medicine. 1999; 341 (7): $527-$ 30.

13. Levine RJ. The need to revise the Declaration of Helsinki. The New England Journal of Medicine. 1999; 341 (7): 531-4.

14. Brody H, Miller FG. The clinician-investigator: unavoidable but manageable tension. Kennedy Institute of Ethics Journal. 2003; 13(4): 329-46.

15. Miller FG, Brody H. A critique of clinical equipoise. Hastings Center Report. 2003; 33(3): 19-28.

16. World Medical Association. Declaration of Helsinki - Ethical principles for medical research involving human subjects. Fortaleza: WMA; 2013.

17. Emanuel EJ. Reconsidering the Declaration of Helsinki. The Lancet. 2013 May 4; 381: 1.532-3.

18. Levine RJ. Op. cit. 1999: 531.

19. Brennan TA. Op. cit. 1999: 529.

20. MacPherson CC. Carta al editor. The New England Journal of Medicine. 1999; 341 (24): 1.852.

21. Levine RJ. Carta al editor. The New England Journal of Medicine. 1999; 341 (24): 1.853.

22. Parsa-Parsi R, Blackmer J, Ehni H-J, Janbu T, Kloiber O, Wiesing U. Reconsidering the Declaration of Helsinki. The Lancet. 2013 out. 20; 382: 1.246-7.

23. Emanuel EJ. Author's reply. The Lancet. 2013 out. 12; 382: 1.247-8.

24. Ndebele P. The Declaration of Helsinki, 50 years later. Jama. 2013. doi:10.100/jama.2013.281316. (acesso 21 out. 2013).

25. Millum J, Wnedler D, Emanuel EJ. The 50th anniversary of the Declaration of Helsinki. Progress but many remaining challenges. Jama. 2013; 310 (20): 2.143.

26. Kottow M. Latinoamérica lesionada. Revista de la Red Latinoamericana de Bioética. 2013; 4 (2): en edición.

27. Kottow M. El ocaso de los placebos. Conferencia y discusión. Florianópolis: X Congreso Brasileño de Bioética; 2013. In: Bioética, saúde, pesquisa e educação. Brasília: CFM; 2014. (no prelo).

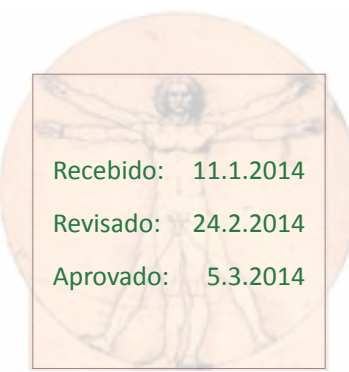

